\title{
Effects of interleukin-1 receptor-associated kinase 1 RNA interference in dendritic cells on inflammatory cytokine release and T-cell proliferation
}

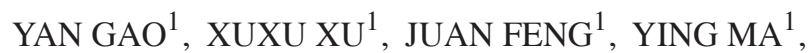 \\ DONGMING ZHENG ${ }^{1}$, YIMING MENG ${ }^{2}$ and FENGPING SHAN ${ }^{2}$
}

\begin{abstract}
${ }^{1}$ Department of Neurology, Shengjing Hospital, Affiliated Hospital of China Medical University, Shenyang, Liaoning 110004;
${ }^{2}$ Department of Immunity, School of Basic Medical Science, Chinese Medical University, Shenyang, Liaoning 110000, P.R. China
\end{abstract}

Received September 21, 2015; Accepted September 14, 2016

DOI: $10.3892 / \mathrm{mmr} .2016 .5946$

\begin{abstract}
The present study aimed to reduce the expression of interleukin-1 receptor-associated kinase 1 (IRAK-1) in dendritic cells (DCs) by RNA interference (RNAi). Subsequently, its effects on the expression of costimulatory surface molecules, the release of inflammatory cytokines, and the proliferation of $\mathrm{T}$ cells during the activation process of DCs, were determined. RNAi was used to silence IRAK-1 gene expression in DCs, followed by lipopolysaccharide stimulation. Flow cytometry was then used to detect the expression levels of DC surface molecules, including cluster of differentiation (CD)86, major histocompatibility complex class II and CD40. Quantitative polymerase chain reaction was conducted to detect the mRNA expression levels of Toll-like receptor 4, IRAK-4, IRAK-1 and nuclear factor- $\kappa \mathrm{B}(\mathrm{NF}-\kappa \mathrm{B})$ in DCs. In addition, ELISA was used to detect the release of the following inflammatory cytokines: Interleukin (IL)-10, IL-12 and tumor necrosis factor- $\alpha$ $($ TNF- $\alpha$ ). The MTS assay was used to determine the effects of IRAK-1 RNAi on T-cell proliferation. Knockdown of IRAK-1 gene expression in DCs significantly reduced the expression levels of costimulatory surface molecules and intracellular $\mathrm{NF}-\kappa \mathrm{B}$, decreased release of the proinflammatory cytokines IL-12 and TNF- $\alpha$, increased release of the anti-inflammatory cytokine IL-10, and significantly reduced the proliferation of $\mathrm{T}$ cells. These results suggested that suppression of IRAK-1 gene expression may inhibit the differentiation and maturation of DCs via the downregulation of DC surface molecules, inhibition of intracellular signal transduction pathways,
\end{abstract}

Correspondence to: Professor Juan Feng, Department of Neurology, Shengjing Hospital, Affiliated Hospital of China Medical University, 36 Sanhao Street, Shenyang, Liaoning 110004, P.R. China

E-mail: juanfengdoc@163.com

Key words: dendritic cells, interleukin-1 receptor-associated kinase 1, toll-like receptor regulation of inflammatory cytokine release, and by reducing its promoting effects on T-cell proliferation.

\section{Introduction}

Natural immunity is the primary line of defense in the resistance of the body against the invasion of foreign pathogens. Induction of the immune response is dependent on recognition and binding of pattern recognition receptors (PRRs), which are found on the surface of natural immune cells, towards the pathogen-associated molecular patterns (PAMPs), thus resulting in activation of signaling pathways and the release of proinflammatory cytokines (1). Toll-like receptors (TLRs) are the most important innate PRRs that mediate the generation of proinflammatory cytokines and initiate the immune response. At present, 14 types of TLR have been identified in mammals, which serve important roles in recognizing foreign antigens, promoting the innate immune response, and promoting activated signaling transduction in cells $(2,3)$.

Although the number of dendritic cells (DCs) is $<1 \%$ of mononuclear cells in peripheral blood, their surface is rich with antigen-presenting molecules [major histocompatibility complex class I(MHC I) and MHC II], costimulatory molecules [cluster of differentiation (CD)80/B7-1, CD86/B7-2, CD40 and CD44] and adhesion molecules [intercellular adhesion molecule (ICAM)-1, ICAM-2, ICAM-3, lymphocyte function-associated antigen (LFA)-1, LFA-3]. Therefore, DCs are considered the strongest professional antigen-presenting cells (APCs) (4). DCs express various PRRs (5), including TLRs, nucleotide-binding oligomerization domain-like receptors, retinoic acid-inducible gene I-like receptors and C-type lectins; these PRRS are able to initiate the transduction of cell signaling and the expression of related genes.

It has been reported that in response to lipopolysaccharide (LPS), TLRs promote interleukin-1 (IL-1)-induced signal transduction in order to mediate activation of other antigen-induced immune cells; this process has been confirmed by TLR2- and TLR4-mediated cellular responses (6). IL-1 receptor-associated kinases (IRAKs) are factors in the TLR/IL-1 receptor (IL-1R) signaling pathway, which has an important role in regulating 
autoimmunity (7). Through kinase and adapter capabilities, IRAKs are able to initiate the inflammatory cascade, and ultimately lead to the expression of immune-associated genes. LPS is able to promote the differentiation of DCs (8), via induction of the TLRs signaling pathway, which regulates the differentiation and function of DCs (9). After binding of LPS to TLR4, regulation of the transcription of various genes occurs via nuclear factor- $\kappa \mathrm{B}(\mathrm{NF}-\kappa \mathrm{B})$ and mitogen-activated protein kinase (MAPK) pathways, thus promoting the maturation of DCs. As a key factor in the TLR/IL-1R signaling pathway, IRAK-1 has an important role in the differentiation and maturation of DCs.

In the present study, RNA interference (RNAi) was performed to silence the gene expression of IRAK-1 in DCs. The present study aimed to determine the underlying mechanisms of IRAK-1 towards the differentiation and maturation of DCs, as well as to investigate its effects on DC physiological functions, including antigen presentation and the promotion of lymphocyte activation.

\section{Materials and methods}

Extraction and culture of DCs. Female C57 BL/6 mice (age, 4 weeks; weight, 13-15 g; specific-pathogen-free level; Experimental Animal Center of Chinese Medical University, Shenyang, China) were selected for use in the present study. The mice were housed with a $12 \mathrm{~h} \mathrm{light} /$ dark cycle, at $22 \pm 2^{\circ} \mathrm{C}$, and free access to food and water. Following intraperitoneal injection of $4 \%$ chloraldurate $(0.01 \mathrm{ml} / \mathrm{g}$ body weight), the mice were sacrificed by cervical spine dislocation in order to obtain the femurs, from which the bone marrow cells were collected and placed into $10 \%$ fetal bovine serum (FBS)-containing 1640 medium (Sigma-Aldrich; Merck Millipore, Darmstadt, Germany). The cells were cultured at $37^{\circ} \mathrm{C}$ in an atmosphere containing $5 \% \mathrm{CO}_{2}$ for $24 \mathrm{~h}$. Subsequently, the supernatant was discarded, $10 \mu \mathrm{g} / \mathrm{ml} \mathrm{IL-4,}$ $10 \mu \mathrm{g} / \mathrm{ml}$ granulocyte-macrophage colony-stimulating factor (GSM-CF) and 10\% FBS-containing 1640 medium were added and the cells were cultured for a further 6 days; half of the medium was changed every $24 \mathrm{~h}$. The supernatant was then discarded and the cells were resuspended in $10 \%$ FBS-containing 1640 medium supplemented with IL-4 and GSM-CF. Subsequently, the cells were counted, re-seeded into 6-well plates $\left(1 \times 10^{6} / \mathrm{ml}\right)$ and cultured at $37^{\circ} \mathrm{C}$ in an atmosphere containing $5 \% \mathrm{CO}_{2}$ for further experimentation. The study was approved by the ethics committee of Shengjing Hospital of China Medical University (Shenyang, China).

Identification and purification of bone marrow mesenchymal stem cells (BMSCs)-originated DCs. The DCs were incubated with anti-CD11c phycoerythrin-conjugated antibody $(50 \mu \mathrm{g} / \mathrm{ml}$; cat. no. 12-0114-82; eBioscience, Inc., San Diego, CA, USA) and isotype matched antibodies for $30 \mathrm{~min}$ at $4^{\circ} \mathrm{C}$. After incubation, cells were washed twice with PBS, and then fixed in $2 \%$ paraformaldehyde (Sigma-Aldrich; Merck Millipore) for $1 \mathrm{~h}$ at $4^{\circ} \mathrm{C}$. Cellular fluorescence was measured by FACScan (BD Biosciences, San Jose, CA, USA), and data were analyzed by FlowJo 7.6.1 software (Tree Star, Inc., Ashland, OR, USA), by calculating the percentage of positive cells compared with the isotype controls. A CD11c MicroBeads sorting kit (Miltenyi
Biotec GmbH, Bergisch Gladbach, Germany) was used to further separate DCs differentiated from BMSCs according to the manufacturer's protocol, and flow cytometry was also used to detect the ratio of $\mathrm{CD} 11 \mathrm{c}^{+}$cells.

$R N A i$. DCs cultured for 6 days and sorted using magnetic beads were subsequently divided into three groups: LPS + mock group, LPS + scrambled small interfering (si)RNA group and LPS + IRAK-1 siRNA group. The LPS + mock group was cultured with IL-4, GSM-CF and 10\% FBS-containing 1640 medium at $37^{\circ} \mathrm{C}$ in an atmosphere containing $5 \% \mathrm{CO}_{2}$ for $24 \mathrm{~h}$; the LPS + scrambled siRNA and LPS + IRAK-1 siRNA groups were transfected with negative control siRNA or IRAK-1 siRNA (Qiagen, Inc., Valencia, CA, USA), respectively, under the same culture conditions as the LPS + mock group. The siRNA groups underwent transfection using HiPerFect transfection kit (Qiagen, Inc.), and were cultured at $37^{\circ} \mathrm{C}$ in an atmosphere containing $5 \% \mathrm{CO}_{2}$ for $24 \mathrm{~h}$. All three groups were cultured for $24 \mathrm{~h}$, and were then treated with $10 \mathrm{mg} / \mathrm{l} \mathrm{LPS}$ (Sigma-Aldrich; Merck Millipore) for $24 \mathrm{~h}$.

Reverse transcription-quantitative polymerase reaction $(R T-q P C R)$. After $24 \mathrm{~h}$ LPS stimulation, the total RNA was extracted from the cells using RNAiso Plus kit (Takara Biotechnology Co., Ltd., Dalian, China) according to the manufacturer's instructions. The reverse transcription reaction was performed using PrimeScript ${ }^{\mathrm{TM}}$ RT kits (Takara Biotechnology Co., Ltd.) according to the manufacturer's instructions. The RT reaction was performed at $37^{\circ} \mathrm{C}$ for $15 \mathrm{~min}$, followed by $85^{\circ} \mathrm{C}$ for $5 \mathrm{sec}$, and the reaction system (20 $\mu 1$ for each sample) was as follows: $4 \mu 1$ PrimeScript Buffer (5X); $1 \mu$ PrimeScript RT Enzyme Mix I; $1 \mu 1$ oligo dt Primer $(50 \mu \mathrm{mol} / \mathrm{l}) ; 1 \mu \mathrm{l}$ random hexamers $(100 \mu \mathrm{mol} / \mathrm{l})$; and $13 \mu l$ total RNA. The PCR system ( $15 \mu$ l for each sample) was as follows: 7.5 $\mu 1$ Premix Ex Taq (2X); $0.25 \mu 1$ forward primer $(10 \mu \mathrm{mol} / \mathrm{l}) ; 0.25 \mu \mathrm{l}$ reverse primer $(10 \mu \mathrm{mol} / \mathrm{l}) ; 3 \mu \mathrm{l} \mathrm{cDNA}$

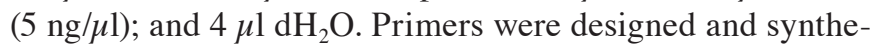
sized by Takara Biotechnology Co., Ltd. (Dalian, China) with $\beta$-actin used as the internal reference gene (Table I). After initial denaturation for $15 \mathrm{~min}$ at $95^{\circ} \mathrm{C}$, the PCR conditions were as follows: For IRAK-1, $95^{\circ} \mathrm{C}$ for $30 \mathrm{sec}, 50$ cycles of $95^{\circ} \mathrm{C}$ for $15 \mathrm{sec}, 61^{\circ} \mathrm{C}$ for $15 \mathrm{sec}$, and $63^{\circ} \mathrm{C}$ for $34 \mathrm{sec}$; for TLR $4,95^{\circ} \mathrm{C}$ for $30 \mathrm{sec}, 40 \mathrm{cycles}$ of $95^{\circ} \mathrm{C}$ for $10 \mathrm{sec}, 58^{\circ} \mathrm{C}$ for $15 \mathrm{sec}$, and $72^{\circ} \mathrm{C}$ for $10 \mathrm{sec}$; for IRAK- $4,95^{\circ} \mathrm{C}$ for $15 \mathrm{sec}$, 40 cycles of $95^{\circ} \mathrm{C}$ for $15 \mathrm{sec}, 65^{\circ} \mathrm{C}$ for $15 \mathrm{sec}$, and $72^{\circ} \mathrm{C}$ for $10 \mathrm{sec}$; for NF- $\mathrm{\kappa B}$ p65, $94^{\circ} \mathrm{C}$ for $30 \mathrm{sec}, 40$ cycles of $95^{\circ} \mathrm{C}$ for $15 \mathrm{sec}, 62^{\circ} \mathrm{C}$ for $15 \mathrm{sec}$, and $70^{\circ} \mathrm{C}$ for $15 \mathrm{sec}$. The relative expression level was determined using the $2^{-\Delta \Delta \mathrm{Cq}}$ analysis method (10).

Flow cytometry. Following 24 h LPS stimulation, DCs in the three groups were collected, washed twice with flow cytometry buffer and adjusted to a cell concentration of $1 \times 10^{6} / 1$. The cells were then incubated with allophycocyanin-CD86 (cat. no. 11-0862), phycoerythrin (PE)-MHC II (cat. no. 11-0367) and PE-CD40 (cat. no. 12-0401) antibodies (eBioscience, Inc.), at final concentrations of $5 \mu \mathrm{g} / \mathrm{ml}$, in the dark at $4^{\circ} \mathrm{C}$ for $30 \mathrm{~min}$. The surface-specific markers of DCs, namely CD86, MHC II and CD40, were then detected using flow cytometry. 
Table I. Primer sequences for quantitative polymerase chain reaction.

\begin{tabular}{lll}
\hline Gene & \multicolumn{1}{c}{ Forward } & \multicolumn{1}{c}{ Reverse } \\
\hline IRAK-1 & CGGACTTCCACAGTTCGAGGTA & TGACCAGCAAGGGTCTCCAG \\
TLR4 & TCACCTCTGCCTTCACTAC & CGTTGGTGCGGTCTATGAG \\
NF- $\mathrm{B}$ p65 & GGATGGCTACTATGAGGCT & CTAATGGCTTGCTC, CAGGTCTC \\
IRAK-4 & ACATGCCCAACGGGTCCTT & ACCTGATGCCATTTGCTGTCCC \\
\hline
\end{tabular}

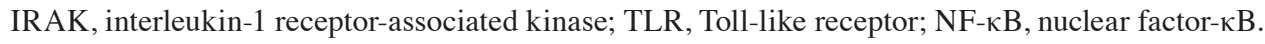

ELISA assay. Following 24 h LPS stimulation, ELISA kits (eBioscience, Inc.) were used to detect IL-10 (cat. no. 88-7314), IL-12 P70 (cat. no. 88-7121) and tumor necrosis factor- $\alpha$ (TNF- $\alpha$; cat. no. 88-7324) in the cell supernatant.

Extraction of T lymphocytes. Female C57 BL/6 mice (age, 4 weeks) were sacrificed and spleens were collected. The spleen samples were placed in PBS and cut into small pieces using scissors. A 200-mesh sieve was then used to gently grind the small spleen sections into a single cell suspension, which was cleared using erythrocyte lysate for $5 \mathrm{~min}$. The suspension was then centrifuged at $300 \times \mathrm{g}$ for $6 \mathrm{~min}$ to discard the supernatant, the cells were resuspended in PBS, and were placed in 10\% FBS-containing 1640 medium for further use.

Sorting of naive $T$ lymphocytes. The Mouse $\mathrm{CD}^{+} / \mathrm{CD} 2 \mathrm{~L}^{+} / \mathrm{CD} 44$ low Naive Column sorting kit (R\&D Systems, Inc., Minneapolis, MN, USA) was used to sort the spleen single cell suspensions, according to the manufacturer's protocol. Naive $\mathrm{T}$ cells that exhibited the expression profile $\mathrm{CD} 4{ }^{+} \mathrm{CD} 62 \mathrm{~L}^{+} \mathrm{CD} 44^{-}$were obtained and cultured in $10 \%$ FBS-containing 1640 medium.

Mixed cell reaction. Following $24 \mathrm{~h}$ LPS stimulation, the cells of the three groups were co-cultured with $\mathrm{CD} 4{ }^{+} \mathrm{CD} 62 \mathrm{~L}^{+} \mathrm{CD} 44$ naive T cells; the ratios of DCs to naive T cells used were 1:1, 1:10, 1:50 and 1:100. After $48 \mathrm{~h}$ co-culture, the MTS assay kit (Promega Corporation, Madison, WI, China) was used to detect the proliferation of naive $\mathrm{T}$ cells according to the manufacturer's protocol. The absorbance at detected at $490 \mathrm{~nm}$.

Statistical analysis. All experiments were repeated three times. The data are presented as the mean \pm standard error of the mean, and were analyzed using SPSS 16.0 statistical software (SPSS Inc., Chicago, IL, USA). Intergroup comparisons were made using one-way analysis of variance and least significant difference tests. Prism version 6.01 (GraphPad Software, Inc., La Jolla, CA, USA) was used to generate the figures. $\mathrm{P}<0.05$ was considered to indicate a statistically significant difference.

\section{Results}

Identification of BMSCs-originated DCs. After BMSCs were cultured in medium containing GSM-CF and IL-4, flow cytometry revealed that the ratio of $\mathrm{CD} 11 \mathrm{c}^{+}$cells was $79.8 \%$ (Fig. 1). Cells obtained from the preliminary culture were then further sorted using magnetic beads, and flow cytometry

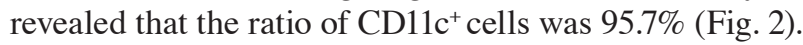

$q P C R$. qPCR was used to detect mRNA expression levels (Fig. 3). Following LPS stimulation, the mRNA expression levels of IRAK-1 in the LPS + IRAK-1 siRNA group were significantly reduced $(\mathrm{P}<0.05$; Fig. $3 \mathrm{~A})$. In addition, the mRNA expression levels of $\mathrm{NF}-\kappa \mathrm{B}$ p65 were decreased in the LPS + IRAK-1 siRNA group ( $\mathrm{P}<0.05$; Fig. 3C). There were no significant differences in the mRNA expression levels of IRAK-1 and NF- $\kappa$ B p65 between the LPS + mock and LPS + scrambled siRNA groups (Fig. 3A and C), thus indicating that IRAK-1 RNAi could reduce its expression in DCs, thus reducing the expression of NF- $\kappa \mathrm{B}$ p65. Furthermore, the mRNA expression levels of TLR4 and IRAK-4 were not significantly different among the three groups ( $\mathrm{P}>0.05$; Fig. $3 \mathrm{~B}$ and $\mathrm{D})$. These results indicate that interfering with the gene expression of IRAK-1 has no significant effects on the mRNA expression levels of the upstream molecules TLR4 and IRAK-4.

Flow cytometry. Following LPS stimulation, flow cytometry was performed to detect MHC II, CD40 and CD86 expression among the three groups. The results demonstrated that the expression levels of MHC II, CD40 and CD86 in the LPS + mock and LPS + scrambled siRNA groups were significantly higher compared with in the LPS + IRAK-1 siRNA group $(\mathrm{P}<0.05)$. However, there was no significant difference between the LPS + mock and LPS + scrambled siRNA groups (Fig. 4). These results indicate that following knockdown of IRAK-1 expression with RNAi, LPS stimulation was able to reduce the expression levels of CD80, MHC II and CD40 in DCs.

ELISA. ELISA kits were used to determine the release of cytokines by DCs (Fig. 5). The results demonstrated that following RNAi-induced knockdown of IRAK-1 gene expression, LPS stimulation was able to significantly reduce the expression levels of the inflammatory cytokines IL-12 and TNF- $\alpha$ (Fig. 5B and C), and promote the expression of the anti-inflammatory cytokine IL-10 (Fig. 5A). There was no difference in the expression levels of IL-12, TNF- $\alpha$ and IL-10 between the LPS + mock and LPS + scrambled siRNA groups; however, when compared with the LPS + IRAK-1 siRNA group, the expression levels of IL-12 and TNF- $\alpha$ were significantly increased, whereas IL-10 expression was significantly reduced. 
Mixed cell reaction. DCs and spleen-originated naive T cells were co-cultured, and cell proliferation was subsequently detected using the MTS assay. The results demonstrated that DCs could significantly promote the proliferation of naive $\mathrm{T}$ cells, which was also associated with the ratio of cell mixtures; the higher the proportion of DCs to naive T cells, the more marked the promoting effects of DCs towards their proliferation (Fig. 6). However, when IRAK-1 gene expression was suppressed by RNAi, the promoting effects of DCs towards the proliferation of naive $\mathrm{T}$ cells were significantly reduced.

\section{Discussion}

Natural immunity is the primary line of defense in the resistance of the body against the invasion of foreign pathogens. PRRs expressed on the surface of natural immune cells are capable of recognizing and binding PAMPs, thus activating the cascade of intracellular signals, promoting the release of inflammatory cytokines and initiating the immune response. TLRs are the most important innate PRRs, which are able to identify pathogens when they are still outside the cell, or have just been phagocytized into inclusion bodies, thus inducing the immune response. Using such signal transduction molecules as IL-1, human TLRs mediate LPS-induced activation of immune cells, such as monocytes. TLRs detect pathogens, and the IL-1R family initiates a rapid cellular response to the released cytokines. IRAKs are an important part of the TLR/IL-1R signaling pathway, which serve an important role in regulating autoimmunity.

Within the IRAKs family, IRAK-1 serves key roles in the IL-1 signaling pathway, and was the first identified IL-1 receptor kinase. Human and murine IRAK-1 were initially cloned in 1996, and Thomas et al demonstrated that the IRAK-1 gene was located at Xq29.52-q 29.7 in rats, and at Xq2810 in humans (11). The molecular weight of IRAK-1 is $\sim 80 \mathrm{kDa}$. Following activation it can be expressed as a $\sim 100 \mathrm{kDa}$ modified form, which via various modifications, including phosphorylation, ubiquitination, acetylation and polyubiquitination, exerts different functions, including interferon regulatory factor (IRF) 5/IRF7, MAPK, and NF- $\mathrm{KB}$ and signal transducer and activator of transcription signaling pathway activation (12-14).

It has been reported that in IRAK-1-deficient macrophages, the expression levels of TNF- $\alpha$ are reduced when TLR2 or TLR4 are activated; this type of TLR-associated damage could weaken the response against the fatal effects of sepsis caused by LPS or gram-negative bacilli (15). Deng et al (16) reported that IRAK-1 ${ }^{-/}$mice are susceptible to a high dose of $S$. aureus compared with wild-type controls. By contrast to the high mortality and extensive weight loss observed in IL-1R-deficient mice in response to $1 \times 10^{6} \mathrm{~S}$. aureus, IRAK-1-1mice are resistant to this low dose of $S$. aureus. Furthermore, IRAK-1 has been shown to serve an important role in TLR7or TLR9-based interferon (IFN) induction; IRAK-1-deficient plasmacytoid DCs could not produce IFN- $\alpha$; however, this deficiency did not affect the generation of other inflammatory factors, such as IL-6 and TNF- $\alpha$ (17). Conversely, previous studies have reported that in IRAK-1-deficient spleen lymphocytes, when TLR9 was activated, the expression levels of

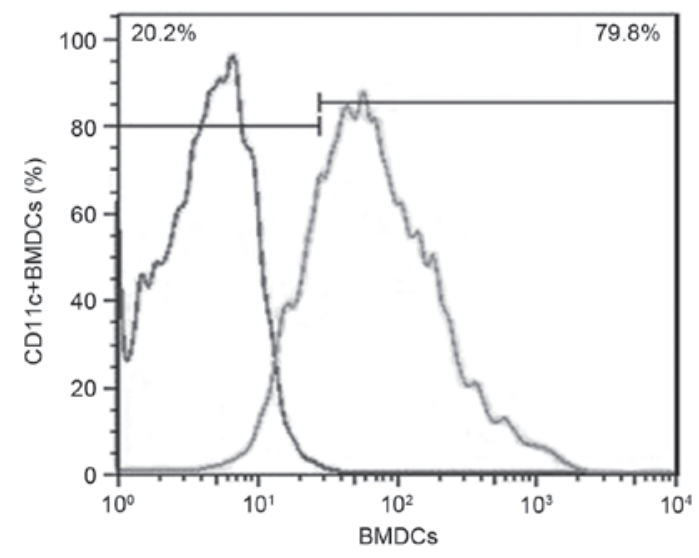

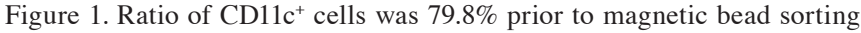
CD11c, cluster of differentiation 11c; BMDcs, bone marrow-derived dendritic cells.

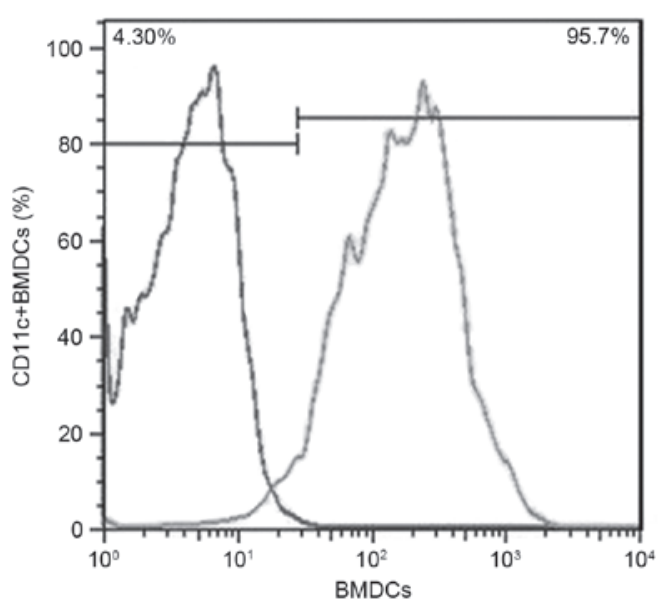

Figure 2. Ratio of CD11c positive cells was $95.7 \%$ after magnetic bead sorting. CD11c, cluster of differentiation 11c; BMDcs, bone marrow-derived dendritic cells.

TNF- $\alpha$ and IL-12 were significantly downregulated (16). These differences may be associated with the different cells selected. Ahmad et al (18) demonstrated that following inhibition of chondrocyte IRAK-1 by RNAi, the expression levels of IL-1 stimulation-induced matrix metalloproteinase 13 (MMP-13) were downregulated, thereby protecting chondrocytes and decreasing the damage caused by MMP-13. These results suggested that IRAK-1 may have clear and necessary roles in TLR7- and TLR9-induced IFN- $\alpha$ downstream induction.

EAE is a T cell-mediated autoimmune disease in mice. The application of myelin protein and complete Freund's adjuvant is able to sensitize mice, resulting in the production of clinical features similar to those observed in human multiple sclerosis. A previous study reported that TLR4 functional defects could weaken activation of T helper (Th)17 and Th1 cells in an EAE model, significantly reducing the symptoms of EAE (19), thus suggesting that TLR4 has a key mediating role in the occurrence of EAE. Furthermore, Deng et al (16) demonstrated in animal studies that IRAK-1-deficient mice were resistant to EAE so that little or no inflammatory response could be detected in the central nervous system of the mice, and the functions of Th1 cells were reduced in such mice. It could be hypothesized that 
A

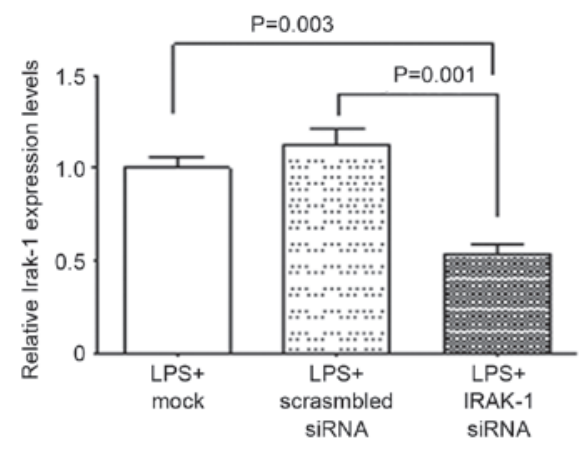

C

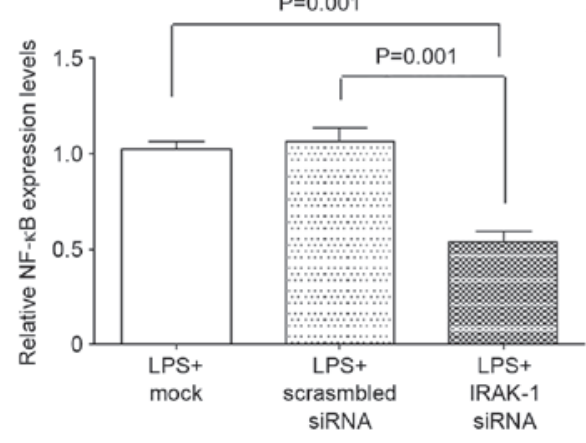

B

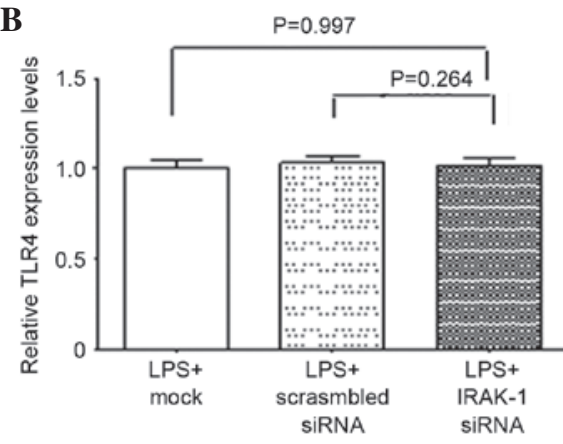

$\mathrm{P}=0.506$

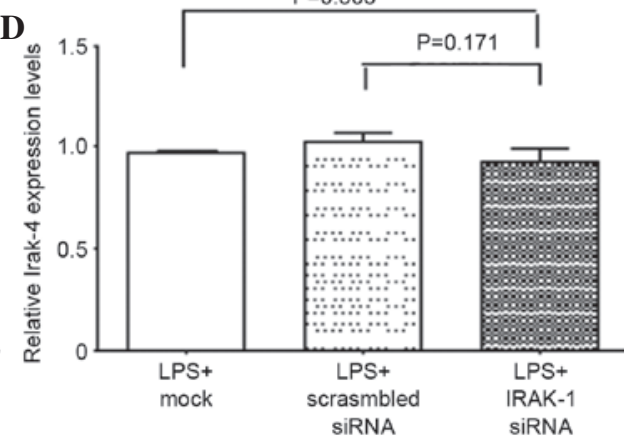

Figure 3. mRNA expression levels of (A) IRAK-1; (B) TLR4; (C) NF-kB p65 and (D) IRAK-4 among the groups. IRAK, interleukin-1 receptor-associated

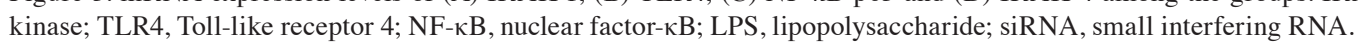
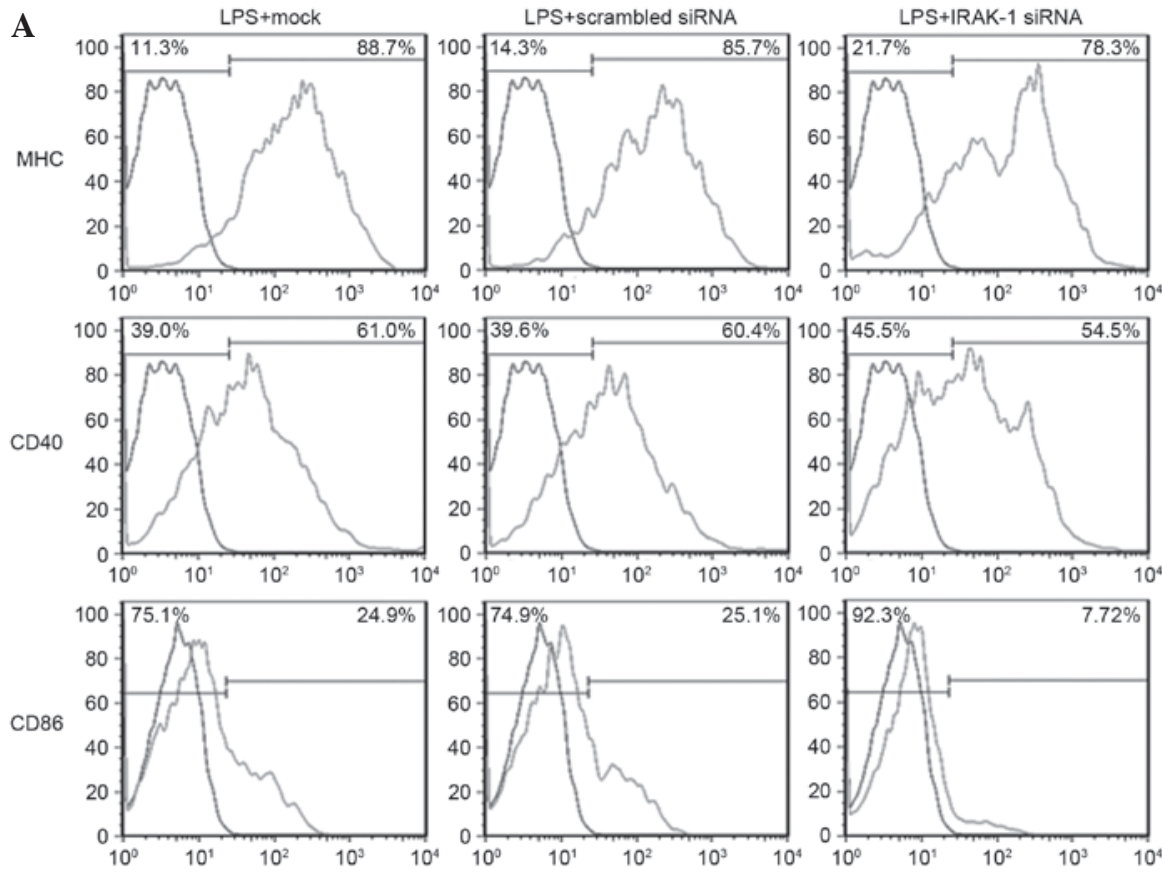

B

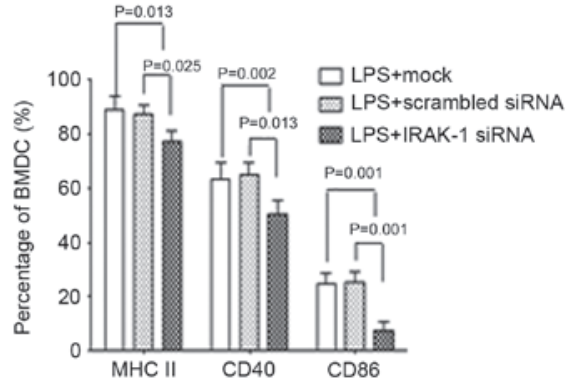

Figure 4. (A) Expression of costimulatory factors among the three groups, as determined by flow cytometry. (B) There were no significant differences between the LPS + mock and LPS + scrambled siRNA groups, whereas the LPS + IRAK-1 siRNA group exhibited a significant reduction in MHC II, CD40 and CD86 IRAK-1, interleukin-1 receptor-associated kinase 1; MHC II, major histocompatibility complex class II; CD, cluster of differentiation; LPS, lipopolysaccharide; siRNA, small interfering RNA; BMDCs, bone marrow-derived dendritic cells. 

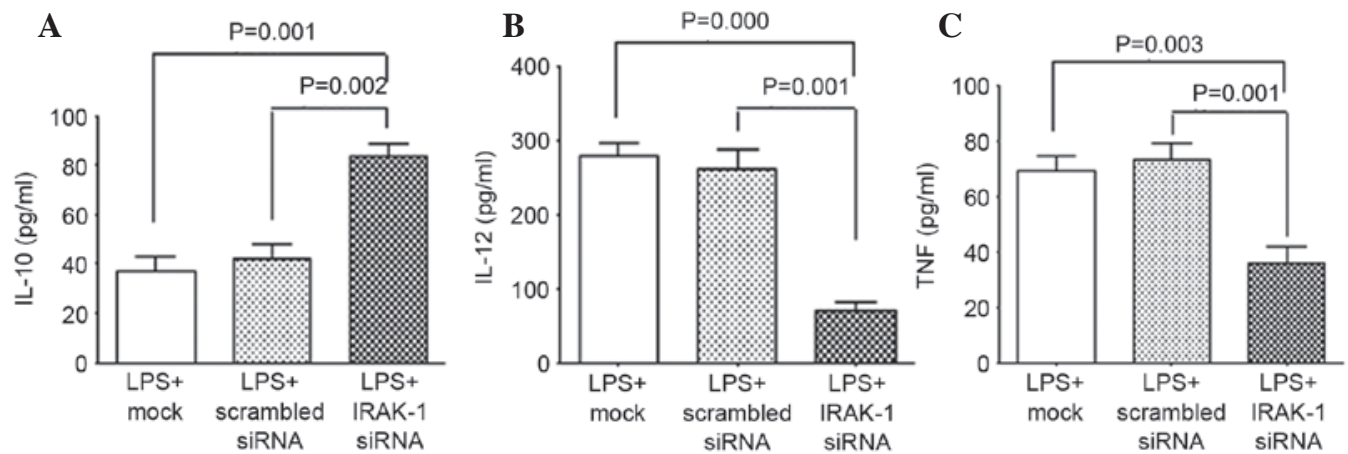

Figure 5. Cytokine expression in the culture supernatant. (A) IL-10; (B) IL-12 and (C) TNF- $\alpha$ expression. IL, interleukin, TNF- $\alpha$, tumor necrosis factor- $\alpha$; IRAK-1, interleukin-1 receptor-associated kinase 1; LPS, lipopolysaccharide; siRNA, small interfering RNA.

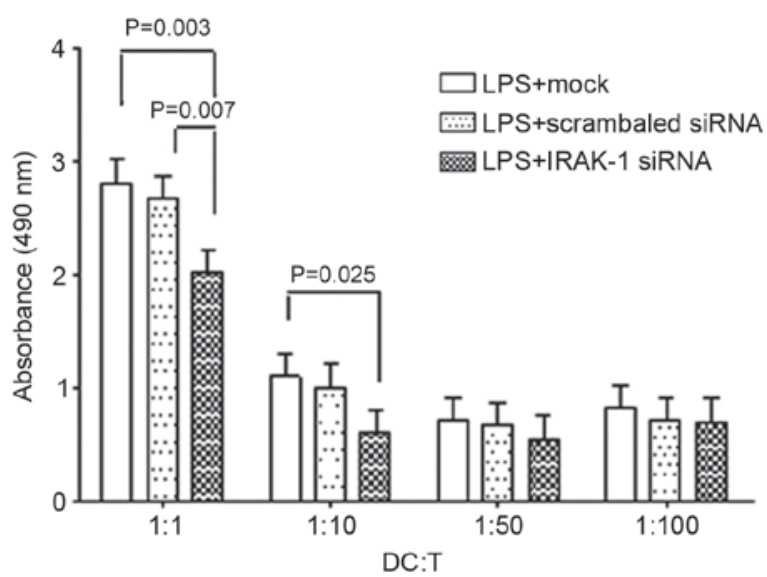

Figure 6. Dendritic cells and naive T cells were co-cultured, and proliferation was determined using the MTS assay. When the proportion of DC to naive T cells was high, the LPS + IRAK-1 siRNA group exhibited weakened promoting effects on the proliferation of naive $\mathrm{T}$ cells. There was no difference between the LPS + mock and LPS + scrambled siRNA groups. IRAK-1, interleukin-1 receptor-associated kinase 1; LPS, lipopolysaccharide; siRNA, small interfering RNA.

the possible reason underlying EAE resistance was due to the suppression of TLR activation during APC-activated IL-1R signaling, which relies on IRAK-1. Other studies have also confirmed that IL-1R- and IL-18-deficient mice appear resistant to the EAE model $(20,21)$. Therefore, it may be speculated that IRAK-1 is associated with EAE; however, the specific mechanisms underlying the effects of IRAK-1 on the occurrence and development of EAE require further investigation.

A previous study reported that the TLR pathway was able to activate the downstream transcription factor NF- $\kappa \mathrm{B}$, thus inducing the expression of various proinflammatory genes (22). IRAK-1 is located downstream of TLR2 in this signaling pathway; therefore, it may serve major roles in signaling transduction from TLR to NF- $\mathrm{kB}$ activation. In the inflammatory responses of various autoimmune diseases, Th17 and Th1 cells serve promoting roles, whereas regulatory $\mathrm{T}$ cells (Treg cells) inhibit this type of regulation; therefore, the responses of Th17 and Th1 cells may be closely associated with the occurrence and development of diseases. IRAK-1 has been confirmed to have key roles in promoting T-cell differentiation towards Th1 and Th17, which may promote the differentiation of Th17 cells and inhibit the generation of Treg cells (23). Therefore, IRAK-1 inhibitors may be considered potential clinical therapeutic tools for the treatment of autoimmune diseases.

The development process of DCs can be divided into two stages: Immature DCs and mature DCs, which exert various functions. Under normal circumstances, the majority of DCs in vivo, which are predominantly contained inside non-lymphoid tissues, are in an immature state. Following ingestion of antigens or stimulation by certain factors (such as LPS, IL-1 $\beta$ and TNF- $\alpha$ ), they may differentiate and become mature. Mature DCs express high levels of MHC molecules, costimulatory molecules and adhesion molecules, integrins (beta-1, beta-2), and characteristic markers (CD1a, CD11c, CD83); secrete cytokines, such as IL-12, IL-21, IL-26, IL-28, TNF- $\alpha$ and IFN- $\alpha$; and stimulate mixed lymphocyte reaction to enhance its abilities. DCs are associated with various autoimmune diseases, and have important roles in the occurrence and development of autoimmune diseases, including multiple sclerosis and its animal model EAE (24). A previous study demonstrated that following pretreatment with LPS and myelin basic protein, the injection of DCs into mice may induce generation of an EAE model (25). Furthermore, a previous study demonstrated that LPS could promote the differentiation of DCs; following the addition of LPS into the DCs culture system, the most typical form of mature DCs appeared after 6 days, the expression levels of mature surface markers CD80 and CD86 were increased, the secretion of IL- 6 and IL-12 were significantly increased, and its roles regarding stimulation of the activation and proliferation of allogeneic T cells were enhanced (8). Another study reported that LPS could promote the maturation of DCs predominantly through the TLRs signaling pathway; after binding with TLR4, LPS may adjust the transcription of various genes via NF- $\kappa B$ and MAPK pathways, thus promoting DC maturation (9). As a key factor in the TLR/IL-1R signaling pathway, IRAK-1 may be involved in the differentiation and maturation of DCs.

Previous studies $(26,27)$ demonstrated that when BMSCs-originated DCs were treated with LPS, IRAK-1 expression was significantly upregulated and NF- $\mathrm{KB}$ was upregulated, thus suggesting that LPS-stimulated maturation of DCs was induced via the TLRs/IL-1Rs pathway. Namely, LPS may bind with TLRs on the surface of DCs, induce the upregulation of IRAK-1, and thus promote the expression of NF- $\kappa B$ and initiation of the immune responses. The present study used RNAi technology to transfect DCs with IRAK-1 siRNA to inhibit the gene expression of IRAK-1. The results 
demonstrated that the differentiation and maturation of DCs were inhibited; flow cytometry revealed that the expression levels of DC surface markers: CD80, MHC II and CD40, were significantly reduced.

IL-10 is a pleiotropic cytokine, the primary function of which is to limit or terminate inflammatory responses. IL-10 is able to regulate the growth and differentiation of several types of cells, including B cells, natural killer cells, cytotoxic T cells, Th cells, monocytes and DCs (28). IL-12 and TNF- $\alpha$ are well-recognized inflammatory factors, which promote inflammatory responses and activate immune cells. Previous studies have suggested that IL-10 has an important role in the occurrence and development of autoimmune diseases, including multiple sclerosis $(29,30)$. IL-10 is able to reduce the expression of IL-12 and TNF- $\alpha$ in various cells, and can downregulate the functions of APCs, thereby regulating the functions of proinflammatory T cells (31). In particular, IL-10 can limit the inappropriate amplification of Th17 cells (32). A previous also demonstrated that IL-10 could inhibit the expression of IL-12 in DCs (33).

The present study demonstrated that following RNAi to silence the expression of IRAK-1 in DCs, the DCs' abilities to release IL-12 and TNF- $\alpha$ were decreased, whereas the expression levels of IL-10 were increased, thus suggesting that the suppression of IRAK-1 could reduce inflammation. In order to further assess the functional alterations of DCs, DCs were co-cultured with naive $\mathrm{CD} 4^{+} \mathrm{T}$ cells. The results demonstrated that the promoting effects of DCs towards the proliferation of $\mathrm{CD}^{+} \mathrm{T}$ cells were significantly inhibited by IRAK-1 siRNA, this inhibition was associated with the ratio of DCs to co-cultured lymphocytes; the higher the ratio, the more obvious the inhibitory effects. These results suggested that IRAK-1 may serve key roles in the differentiation and maturation of DCs.

In conclusion, during LPS-mediated differentiation and maturation of DCs, IRAK-1 may serve key roles. Following the suppression of IRAK-1, the differentiation and maturation of DCs was inhibited in several ways; IRAK-1 RNAi reduced the expression of cell surface factors CD86, CD40 and MHC II; reduced mRNA expression of $\mathrm{NF}-\kappa \mathrm{B}$ p 65 ; reduced the expression of proinflammatory cytokines IL-12 and TNF- $\alpha$, and enhanced the expression of the anti-inflammatory cytokine IL-10. In addition, the promoting abilities of DCs towards the activation and proliferation of $\mathrm{T}$ cells were reduced. Following the completion of further studies regarding the mechanisms of IRAKs in innate immune processes, novel drugs and methods may be identified for the clinical treatment of autoimmune diseases, such as systemic lupus erythematosus and multiple sclerosis, thus advancing the diagnosis and treatment of human autoimmune diseases.

\section{Acknowledgements}

The present study was supported by the Projects of Liaoning Provincial Science and Technology Department (grant no. 2012225021) and Program of Basic and Clinical Research Platform of China Medical University (grant no. CMU201406).

\section{References}

1. Kumar H, Kawai T and Akira S: Pathogen recognition by the innate immune system. Int Rev Immunol 30: 16-34, 2011.
2. Fischer $M$ and Ehlers M: Toll-like receptors in autoimmunity. Ann N Y Acad Sci 1143: 21-34, 2008.

3. Brown J, Wang H, Hajishengallis GN and Martin M: TLR-signaling networks: An integration of adaptor molecules, kinases and cross-talk. J Dent Res 90: 417-427, 2011.

4. Satpathy AT, Wu X, Albring JC and Murphy KM: Re(de)fining the dendritic cell lineage. Nat Immunol 13: 1145-1154, 2012.

5. Pearce EJ and Everts B: Dendritic cell metabolism. Nat Rev Immunol 15: 18-29, 2015.

6. Xiong Y, Qiu F, Piao W, Song C, Wahl LM and Medvedev AE: Endotoxin tolerance impairs IL-1 receptor-associated kinase (IRAK) 4 and TGF-beta-activated kinase 1 activation, K63-linked polyubiquitination and assembly of IRAK1, TNF receptor-associated factor 6 and IkappaB kinase gamma and increases A20 expression. J Biol Chem 286: 7905-7916, 2011.

7. Cohen P: The TLR and IL-1 signalling network at a glance. J Cell Sci 127: 2383-2390, 2014.

8. Mihret A, Mamo G, Tafesse M, Hailu A and Parida S: Dendritic Cells activate and mature after infection with mycobacterium tuberculosis. BMC Res Notes 4: 247, 2011.

9. Zanoni I, Ostuni R, Capuano G, Collini M, Caccia M, Ronchi AE, Rocchetti M, Mingozzi F, Foti M, Chirico G, et al: CD14 regulates the dendritic cell life cycle after LPS exposure through NFAT activation. Nature 460: 264-268, 2009

10. Livak KJ and Schmittgen TD: Analysis of relative gene expression data using real-time quantitative PCR and the 2(-Delta Delta C(T)) Method. Methods 25: 402-408, 2001.

11. Thomas JA, Allen JL, Tsen M, Dubnicoff T, Danao J, Liao XC, Cao Z and Wasserman SA: Impaired cytokine signaling in mice lacking the IL-1 receptor-associated kinase. J Immunol 163: 978-984, 1999.

12. Suzuki N, Suzuki S, Duncan GS, Millar DG, Wada T, Mirtsos C, Takada H, Wakeham A, Itie A, Li S, et al: Severe impairment of interleukin-1 and Toll-like receptor signalling in mice lacking IRAK-4. Nature 416: 750-756, 2002.

13. Kim TW, Staschke K, Bulek K, Yao J, Peters K, Oh KH, Vandenburg Y, Xiao H, Qian W, Hamilton T, et al: A critical role for IRAK4 kinase activity in Toll-like receptor-mediated innate immunity. J Exp Med 204: 1025-1036, 2007.

14. Beinke S, Robinson MJ, Hugunin $M$ and Ley SC: Lipopolysaccharide activation of the TPL-2/MEK/extracellular signal-regulated kinase mitogen-activated protein kinase cascade is regulated by I kappa B kinase-induced proteolysis of NF-kappa B1 p105. Mol Cell Biol 24: 9658-9667, 2004.

15. Swantek JL, Tsen MF, Cobb MH and Thomas JA: IL-1 receptor-associated kinase modulates host responsiveness to endotoxin. J Immunol 164: 4301-4306, 2000.

16. Deng C, Radu C, Diab A, Tsen MF, Hussain R, Cowdery JS, Racke MK and Thomas JA: IL-1 receptor-associated kinase 1 regulates susceptibility to organ-specific autoimmunity. J Immunol 170: 2833-2842, 2003.

17. Uematsu S, Sato S, Yamamoto M, Hirotani T, Kato H, Takeshita F, Matsuda M, Coban C, Ishii KJ, Kawai T, et al: Interleukin-1 receptor-associated kinase-1 plays an essential role for Toll-like receptor (TLR)7- and TLR9-mediated interferon-alpha induction. J Exp Med 201: 915-923, 2005.

18. Ahmad R, Sylvester J and Zafarullah M: MyD88, IRAK1 and TRAF6 knockdown in human chondrocytes inhibits interleukin-1-induced matrix metalloproteinase-13 gene expression and promoter activity by impairing MAP kinase activation. Cell Signal 19: 2549-2557, 2007.

19. Reynolds JM, Martinez GJ, Chung Y and Dong C: Toll-like receptor 4 signaling in $\mathrm{T}$ cells promotes autoimmune inflammation. Proc Natl Acad Sci USA 109: 13064-13069, 2012.

20. Schiffenbauer J, Streit WJ, Butfiloski E, LaBow M, Edwards C III and Moldawer LL: The induction of EAE is only partially dependent on TNF receptor signaling but requires the IL-1 type I receptor. Clin Immunol 95: 117-123, 2000.

21. Shi FD, Takeda K, Akira S, Sarvetnick N and Ljunggren HG: IL-18 directs autoreactive T cells and promotes autodestruction in the central nervous system via induction of IFN-gamma by NK cells. J Immunol 165: 3099-3104, 2000.

22. Doyle SL and O'Neill LA: Toll-like receptors: From the discovery of NFKB to new insights into transcriptional regulations in innate immunity. Biochem Pharmacol 72: 1102-1113, 2006.

23. Maitra U, Davis S, Reilly CM and Li L: Differential regulation of Foxp3 and IL-17 expression in CD4 T helper cells by IRAK-1. J Immunol 182: 5763-5769, 2009.

24. Galicia G and Gommerman JL: Plasmacytoid dendritic cells and autoimmune inflammation. Biol Chem 395: 335-346, 2014. 
25. Mellanby RJ, Cambrook H, Turner DG, O'Connor RA,Leech MD, Kurschus FC, MacDonald AS, Arnold B and Anderton SM: TLR-4 ligation of dendritic cells is sufficient to drive pathogenic $\mathrm{T}$ cell function in experimental autoimmune encephalomyelitis. J Neuroinflammation 9: 248, 2012.

26. Zhang S, Yang N,Ni S, Li W, Xu L, Dong P and Lu M: Pretreatment of lipopolysaccharide (LPS) ameliorates D-GalN/LPS induced acute liver failure through TLR4 signaling pathway. Int J Clin Exp Pathol 7: 6626-6634, 2015.

27. Read MA, Cordle SR, Veach RA, Carlisle CD and Hawiger J: Cell-free pool of CD14 mediates activation of transcription factor NF-kappa B by lipopolysaccharide in human endothelial cells. Proc Natl Acad Sci U S A 90: 9887-9891, 1993.

28. Tian G, Li JL, Wang DG and Zhou D: Targeting IL-10 in auto-immune diseases. Cell Biochem Biophys 70: 37-49, 2014

29. Hesse D, Krakauer M, Lund H, Søndergaard HB, Limborg SJ, Sørensen PS and Sellebjerg F: Disease protection and interleukin-10 induction by endogenous interferon-beta in multiple sclerosis? Eur J Neurol 18: 266-272, 2011.
30. Correa F, Hernangómez-Herrero M, Mestre L, Loría F, Docagne $\mathrm{F}$ and Guaza $\mathrm{C}$ : The endocannabinoid anandamide downregulates IL-23 and IL-12 subunits in a viral model of multiple sclerosis: Evidence for a cross-talk between IL-12p70/IL-23 axis and IL-10 in microglial cells. Brain Behav Immun 25: 736-749, 2011.

31. O'Garra A and Murphy KM: From IL-10 to IL-12: How pathogens and their products stimulate APCs to induce T $(\mathrm{H}) 1$ development. Nature Immunol 10: 929-932, 2009.

32. Chaudhry A, Samstein RM, Treuting P, Liang Y, Pils MC, Heinrich JM, Jack RS, Wunderlich FT, Brüning JC, Müller W and Rudensky AY: Interleukin-10 signaling in regulatory T cells is required for suppression of Th17 cell-mediated inflammation. Immunity 34: 566-578, 2011.

33. Ruffell B, Chang-Strachan D, Chan V, Rosenbusch A, Ho CM, Pryer N, Daniel D, Hwang ES, Rugo HS and Coussens LM: Macrophage IL-10 blocks CD8 ${ }^{+} \mathrm{T}$ cell-dependent responses to chemotherapy by suppressing IL-12 expression in intratumoral dendritic cells. Cancer Cell 26: 623-637, 2014. 